Original Research Paper

\title{
Influence of Time, Temperature and Pressure on the Creasing of Wool/Polyester Blended Fibers
}

\author{
Brian F.G. Katz \\ Sorbonne Université, CNRS, Institut Jean Le Rond d'Alembert, UMR 7190, Paris, France
}

Article history

Received: 06-10-2021

Revised: 24-11-2021

Accepted: 29-11-2021

Email: brian.katz@sorbonne-universite.fr

\begin{abstract}
Studies investigating the response of a natural/synthetic blended material to variations of time, temperature and pressure with respect to induced deformations have been carried out. Such a material is interesting due to its complexity of responses which are dependent on the nature of each fiber. The study's sample was a wool/polyester blend with a 45/55 composition respectively. This combination is a common material and is resistant to deformations. The application of a quantitative systematic approach provides substantial correlation between combinations of conditions that would not be evident in a more common qualitative approach. Strict adherence to a quantitative method makes this investigation more informative than existing research literature. Pressure and temperature ranges corresponded to values associated with clothing presses. The deformations were evaluated by examining the crease formed and associating a corresponding crease value. Analysis of the crease values and parameters established a clear correlation between the conditions and the resulting crease values. This case study shows that time and temperature are key factors in the creasing of materials, with pressure slight less influential in determining the maximum deformation possible. A regression model was determined to predict the resulting "crease angle" as a function of these parameters, representing $87 \%$ of the variance of the results. This experimental methodology can be generalized and applied to alternate fabrics to adapt the corresponding model.
\end{abstract}

Keywords: Deformation, Creasing, Blended Fabric, Predictive Model

\section{Introduction}

This investigation is part of a general interest in the mechanical properties of textiles and methods for imparting deformations within them. Research into deformations within fiber materials is a relatively unexplored field with sporadic work carried out dealing with particular textile designs and specific points of interest. Previous work has looked in to the effects of various conditions on either the introduction or removal of semi-permanent deformations, commonly known as a "crease". The response of fibrous materials to the conditions of time, temperature, pressure and humidity are of interest to the textile and garment industries as a whole. Little work has been done to correlate specific conditions to material response. The majority of published research has dealt with random parameters and their effects.

The aim of this constrained investigation was to examine the effects of time, temperature and pressure on the mechanical properties of textile materials in a controlled experiment. This differed from previous works in that a systematic experiment is used resulting in quantitative results for each parameter. It was hoped that this investigation would lead to insight into the determining factors involved in the deformation of a fibrous material. In order to do this, a physical parameter was defined, measured and correlated to the specific condition parameters.

\section{Literature Survey}

\section{Deformation Experiments}

A number of investigations have been carried out concerning the deformation of fabrics, in particular wool/polyester blends. What is most striking about the various experiments is the range of parameters that have been used and the different quantities actually measured. Each measurement conveys information crucial to the experiment, but it is sometimes difficult to relate to other experiments. 
Leeder (1975) performed experiments on wool and wool/polyester fabrics in relation to temperature. The actual measurements involved the magnitude and subsequent decay of a deformation after a given time period. What was shown was that greater temperatures induced more severe deformations. In addition, wool/polyester blended fibers resisted deformations and recovered induced deformations quicker than $100 \%$ wool fibers.

Under normal atmospheric conditions (defined as mild, ambient) involving minimal changes in temperature and humidity, there appeared to be no real discernible difference between wool and wool/polyester fabric response (Looney and Handy, 1968; Loasby et al., 1956). What must be emphasized though are the conditions under which the experiment took place. Temperatures ranged from $20^{\circ} \mathrm{C}$ to $35^{\circ} \mathrm{C}$ with times being between $30 \mathrm{~min}$ and 8 days. Humidity levels throughout the experiment varied from 0 to $100 \%$ RH. There is no real mention of the exact materials used, their conditions, or the experimental apparatus.

Several experiments were conducted with respect to the deformation resistance of several synthetic fibers. Though there was a great deal of difference between the fibers, there was a comment by Skelton (1968) saying that the results "of varying magnitude" depended "on the viscoelastic nature of the material, the speed at which the press is opened and the detailed geometry of the crease." This disclaimer makes detailed analysis and inspection of the data very difficult.

Somewhat similar experimental work was performed by Ranganathan et al. (1971). An analysis of crease recovery as it related to moisture content and temperature was carried out. In this case, experiments were performed on a cotton/polyester blended fiber. The material used was precisely a " $67 \%$ Terylene [form of polyester] $33 \%$ cotton, having $49 \mathrm{ends} / \mathrm{cm}^{2}$ and $25 \mathrm{picks} / \mathrm{cm}$ of 14 -tex yarns; its weight was $103 \mathrm{gm}^{-2}$ and it was chlorine-bleached, mercerized and heat-set" (Ranganathan et al., 1971, p. 393). This type of detailed description of the material was used by several researchers.

Each one though described their material slightly differently using different parameters. An example of another method for material description is: "plain weave with 17 ends and picks/cm of 30 tex X 2 yarns of 64's quality wool: it was finished with $10 \mathrm{~min}$ of crabbing. There was a colored yarn every 100 ends and picks" (Ly and Denby, 1983). It is, in general, difficult to know so precisely the content and history of the material used as Ranganathan et al. (1971) were able to do. Usually only the textile manufacturer knows these details. Various researchers give varying degrees of information about their material samples from ones as complicated as these to simply 'a wool/polyester blend'.

It is worth mentioning that several interesting points came out of the research of Ranganathan et al. (1971) An analysis of the effects of resin treatments with respect to moisture humidity and crease-recovery showed that results were quite sporadic from 0 to $15 \% \mathrm{RH}$ and then began to stabilize into some simple curve. These measurements were made at $20^{\circ} \mathrm{C}$. Loads of $1 \mathrm{~g} /$ thread and $5 \mathrm{~g} /$ thread were used to induce the creases. The temperature range for the experiments was from $-10^{\circ} \mathrm{C}$ to $110^{\circ} \mathrm{C}$, with a different humidity level at each temperature. This unsystematic method of measurements makes any serious analysis and comparison difficult, since no two measurements were fixed with respect to both temperature and humidity. What is interesting to note is that there was a sharp decline in crease-recovery properties (equated to a rise in crease angle as defined in the current study a.k.a residual angle Skelton, 1968) in the region between $50-70^{\circ} \mathrm{C}$. This effect was not present in measurements performed on pure cotton fabrics. It is therefore possible to postulate that this effect is due to the polyester content of the fibers. Work done regarding strain recovery as pertaining to processing techniques was carried out by Postle et al. (1988). The effects of various dyeing techniques on the thermal stability and strain recovery properties were also investigated. Additional work regarding the methods of fiber production and cleaning solutions were included, with all of these different parameters seen to have a noticeable effect on the physical performance of the fiber material.

\section{Deformation Theory}

A large amount of theoretical work has been presented by Skelton (1968). The main work deals with an attempt to derive a mathematical solution for single component material deformations, specifically crease recovery. For simplicity, calculations were restricted to creases parallel to the thread direction. As noted by the study's author, this limitation in the calculation was restrictive to the point that the effect of two crossing threads perpendicular to the crease line results in interference severe enough to invalidate any predictive result. The calculations contained a vast number of parameters, including tensile elastic recovery performance, stress/strain relations, twist, crimp, dimensions, material finishes (both chemical and physical) and material compensation coefficients.

The variables for different materials vary significantly enough that there is separate data for each region of material production and also for the slight differences in polymer production methods. American cotton is attributed slightly different coefficients than Australian cotton. Various densities of polyester exist are listed (e.g., 1.5 den., 3 den., 4.5 den. fibers) each having different coefficients and parameter values. Comparisons between calculated and experimental values for the crease recovery angle (c.r.a. = $180^{\circ}$-crease_angle) resulted in errors of $\pm 5 \%$ for wool fabrics and $\pm 10 \%$ for polyester materials (Skelton, 1968). No data for all the variables was found in the cited report.

Less numerical analysis of deformations in both wool and polyester has been performed. The majority of this study deals with the actual fiber mechanics, as pertaining to the architecture and assembly within the material. Analysis of the knit structure and twist pattern as they 
relate to material strength exists for both wool (Smuts, 1989, p. 8192) and polyester (Militký et al., 1991, pp. 80-86, 90-98, 196-201) fibers.

A very complicated analysis of weave patterns and knit structures was carried out by Postle et al. (1988, p. 150) with complex equations for different loop structures developed to deal with fabric extension and deformation processes. For the major portion of their analysis though, a fabric unit cell consisting of a single knitted loop was used for simplicity (Postle et al., 1988, p. 163). Additional work concentrated on the binding patterns of blended fibers. This can be done in a number of ways, with different fibers combined around one fiber as a core, or braided around a vacant core, or produced in several helix patterns, each having very different effects of the physical properties of the fibers and subsequently the materials they are used in.

\section{Current Study}

Prior studies were seen to be limited in their control of experimental conditions and the use of a reliable metric for rapidly quantifying the deformation. As such, the main goal of the current investigation was to quantify crease evaluation as a function of deformation conditions and concentrate more on objective rather than subjective measurements. It is further intended to provide a predictive model, based on experimental results, that requires less extensive material property details while providing comparable performance to more physically based theoretical models.

\section{Measurements}

A protocol for deformation measurements was developed. A deformation in material results in an apparent and observable crease. It is obvious from cursory examination that there are degrees of creaseness, which could be qualified as say, a strong crease or a weak crease. Previous studies involving the creasing of material have used a simple ranking system for the crease value (Kojima, 1986). Creases were evaluated by assigning a Crease Class ranging from 1-5, with Classes defined as: No crease, faint crease, no sharp crease, faintly sharp crease and very sharp crease.

Several general methods for evaluating creases exist (Smuts, 1989; Hunter and Fan, 2015). One procedure involves determining the height of the crease with respect to the bulk material. Another means of crease measurement is based upon a set of wedges which are placed within the formed crease. The wedge that corresponds best to the shape of the deformation is noted and the crease valued. A third method is to take the material and place it on a rotating plate and record the angle of deviation from a linear path. There are a large number of variations on these schemes which concentrate more on the specific performance factor in question or around different material theories (Smuts, 1989, p. 133-4). Since these varying methods are controlled differently and function differently, some inconsistencies relating to specific conditions or material properties are ignored or enhanced resulting in anomalies between methods (Smuts, 1989, p. 149).

Looking at the various methods there are several factors that resulted in the selection of one over another. Measuring the crease height, though simple, has a large possibility for error due to the small physical property actually being measured. True changes in the material response would be difficult to distinguish from material and experimental anomalies. The wedge method is based on the design and manufacture of a large set of crease standards. The accuracy of this method is dependent upon the number and precision of the crease wedges. The rotating disc, or Shirley crease recovery tester ${ }^{1}$ (Choudhury, 2017), method requires the construction of a single measuring apparatus of relatively simple concept. The disc method is also quicker, resulting in more accurate measurements being made before the deformation relaxes. The time for each measurement is also relatively constant, since different measurements only require rotations of a plate, rather than repetitive guesses until the correct wedge is selected. For these reasons the rotating disc method was used in this investigation.

Crease measurements are then made for a wide range of conditions. The material is subjected to condition under various exposure times, temperatures and pressures, with the corresponding crease value recorded. The results of the measurements are values that can be compared for the different conditions.

\section{Experiment}

The experiment comprised a series of measurements over increasing temperature at various conditions, with either fixed time or fixed pressure. The result was a four-dimensional matrix representing time, temperature and pressure as they related to the crease angle. The specific range of conditions encompasses the various environments that textile materials are typical subjected to where deformations are concerned. The temperature and pressure ranges are comparable to those used by irons and clothing presses.

\section{Materials}

A single material sample was chosen for this study, as studies have shown that creasing is dependent on construction details of both the fiber and material (Choudhury, 2017). The choice of materials open to analysis was extensive. In choosing the test material several considerations were examined. The response of

\footnotetext{
${ }^{1}$ https://textilestudycenter.com/crease-resistance-and-recovery-test/
} 
the material to the experimental conditions needed to be adequate enough to result in a full spectrum of measurements. Certain materials tended to deform quite easily and produce crease values only at the high end of the scale given the range of conditions considered. In addition to the response of the material was the interest value. Materials that have a reputation for being difficult to crease would provide interesting insight into what was required to obtain sufficient creases and this was considered.

General material properties are well known (Skelton, 1968). Cotton fibers wrinkle easily (Mercer and Moore, 1975a; Choudhury, 2013); wool material does not hold a press well (Hosseini Ravandi and Valizadeh, 2011); and polyester is quite difficult to crease (Mercer and Moore, 1975b). Given that these are common materials it seemed appropriate to consider them, rather than other exotic materials. Since we were interested in the response over a wide range of conditions, a material than did not conform easily was desirable. This consideration resulted in a choice between wool and polyester materials. It was this combination of natural and synthetic fibers that had the possibility to be the most interesting and useful, since the blend of wool and polyester is a commonly used fabric and also is known to be resistant to deformations (Mercer and Moore, 1975b). The material selected was a wool/polyester blend (45/55 composition), the major percentage being polyester as this is more difficult to crease.

\section{Experimental Apparatus and Instrumentation}

\section{Deformation Induction}

Material deformations were carried out using a set of brass weights and an electric hotplate (Stuart, model no. SM3). A brass plate was placed on the surface of the hotplate to provide a clean, smooth surface of equal thermal conductivity as the sample weights. The temperature of the plate was controlled externally.

\section{Thermal Control}

Temperature was monitored using a basic copper-constantan thermocouple. A reference junction at $0^{\circ} \mathrm{C}$ (ice water) provided the basis for the measurements. A computer-controlled feedback monitoring circuit was used to control the heating element, resulting in a sample temperature accurate to $\pm 0.5^{\circ} \mathrm{C}$. A mercury thermometer was also attached to the hotplate for temperature monitoring.

\section{The Creasometer}

The creasometer was based on a Shirley Crease Recovery Tester, comprising a sample holder, crease scale, alignment point and uni-axle free rotating mount. A schematic of the device is shown in Fig. 1, while full details can be found in Gray (1992). The sample holder is a fixed plate with a spring mounted arm used to secure the sample. The ends of the plate and arm coincide in order to position the crease correctly with regards to the scale. The arm holds the sample flush against the plate ensuring that the deformation force acts only upon the suspended section of the sample and that no motion occurs within the sample holder.

Studies in Skelton (1968) concerning crease recovery were based upon a slightly different creasometer design, where the sample holder was shortened so that the deformation point was at a distance of $0.16 \mathrm{~cm}$ from the sample holder clamp. This resulted in more curvature at the deformation point, with results less comparable to other experiments. For comparison to the current study, we note that Skelton creased samples for $1 \mathrm{~min}$ and then allowed them to relax for $1 \mathrm{~min}$ before measurements were made, some being under $1 \mathrm{lb} / \mathrm{in}^{2}$ and others at $10 \mathrm{lb} / \mathrm{in}^{2}$, with environmental conditions of $20^{\circ} \mathrm{C}$ and $65 \% \mathrm{RH}$.

Crease angle measurements are made with reference to the angular graded scale. The scale has its center at the point of deformation. The alignment point is a half-cylinder rod mounted vertically with the flat face perpendicular to the scale and coincidental with the deformation point. The rod is mounted external to the rotating mount. The scale is calibrated with respect to the mounting plate, given that a "no crease" case would result in the sample being parallel with the rod.

\section{Deformation Removal}

In order to make repeated measurements upon the same sample, it was necessary to remove the deformation after each measurement. This ensured that there was no interference from previous material handling. A method was adopted using a steam iron (Morphy Richards, model no. 42065). The thermostat was set to $50 \%$, roughly equivalent to $110^{\circ} \mathrm{C}$. The temperature was kept at this low value to insure there was no damage or modification to the sample through polymer reactions of the material. Steam was not used unless necessary, that being after high temperature deformations where the crease was greater. An additional piece of material was placed between the iron and the samples to reduce the amount of heat damage and wear to the sample.

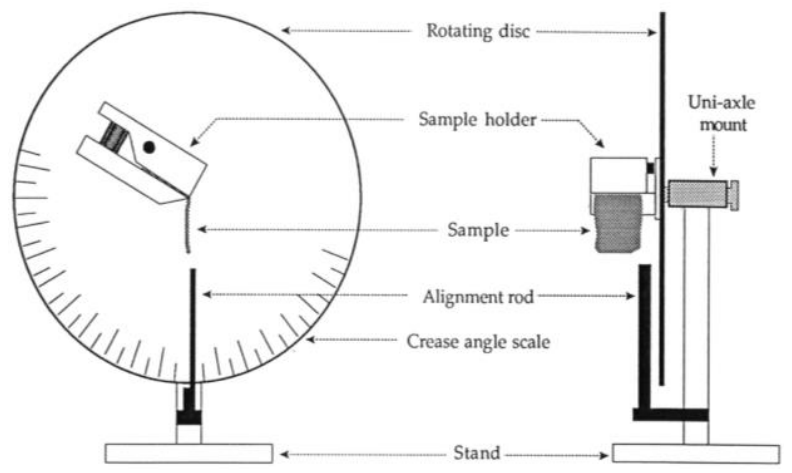

Fig. 1: Schematic of the creasometer 


\section{Sample Materials}

Material used for the experiment was taken from a pair of Trent trousers with a fiber composition of 55\% polyester and $45 \%$ wool. Sections of material were removed from the trousers and cut into $3 \times 6 \mathrm{~cm}$ samples such that the weave of the material was at $45^{\circ}$ to the cut edges. Boundary conditions of the sample were left as raw cut edges, without hemming or other treatment, so as to provide an example of the basic material simple

\section{Experimental Methods}

\section{Basic Methods}

Each set of data comprises a series of measurements recorded at a specific pressure and time with varying temperature. The same sample is used for the entire data set. This is to ensure continuity within the data set and remove any possible errors that may be due to irregularities between samples. Each data set actually contains data taken for three samples. Measurements are made in the vicinity of a central temperature for each sample group. These temperatures varied by $2^{\circ} \mathrm{C}$ to $3^{\circ} \mathrm{C}$, so that measurements at $50^{\circ} \mathrm{C}$ would be taken at $48^{\circ}, 50^{\circ}$ and $52^{\circ}$.

The result of such an approach is to achieve a sense of continuity of measurements and allow for sample irregularities. The use of three samples also provided a foundation for reproducibility. Measurements are made until the samples' properties are altered from their initial conditions. This corresponds to either permanent deformations or structural changes within the material. Permanent deformations are recognized when, after several attempts at deformation removal with limited amounts of steam, the deformation persists. Excessive temperatures and steam are not used, since this could result in structural changes within the sample, resulting in a change in the feel and texture of the sample; it becoming smoother and stiffer. When either of these changes occurs within a sample group the data set is concluded.

\section{Procedure}

All samples were ironed once, without steam, before the experiment. Each measurement began by instructing the computer on the desired experimental temperature. After this temperature was achieved with minimal drift, the sample was placed upon the deformation inducer's brass plate. The sample was lightly folded with the edges held in place with a pair of tweezers. The brass weight was then slowly slid along the tweezers until it covered the sample, at which point the tweezers were removed and the timer activated. The reason for such care was to ensure that the sample remained folded along the center and that when the weight was placed the alignment of the edges was not altered and the fibers were not distorted (Raes et al., 1974).

When the experimental time expired the weight was removed and the sample was retrieved using a pair of tweezers. The sample holder was opened and the sample carefully placed inside with the deformation point lying along the edge of the sample holder. The sample holder was closed and rotated until the external section of the sample hung vertically, in-line with the alignment point. The crease angle was then recorded. After each of the three samples were measured, they were all placed upon a surface and covered with another piece of material. The deformations were then removed using the deformation removal procedure. Smooth, even pressure was applied to the samples. The samples were then turned over and the process was repeated. If the deformations remained, the process was repeated again. If they remained, the iron was set to release a burst of steam for no more than $3 \mathrm{~s}$ and the process was repeated. If the deformation still remained at this point, it was considered to be permanent and the data set was deemed complete. Once the deformations were removed, any stray material that had become loose during the deformation formation and removal procedures was trimmed using a pair of scissors. Total loss in mass was $\leq 3 \%$ per sample across the entire measurement session. The entire procedure was then repeated for the next set of measurements.

\section{Experimental Precautions and Errors}

There are several areas where errors could occur within this experiment. Some of these are due in part to the very nature of the experiment and sample material. Some of the errors can be expected, anticipated and even removed, but others remain regardless. Several methods have been devised to limit the number of errors in measurement and subsequent analysis.

\section{Sample Handling}

It is very important how the samples are treated during the deformation and measurement processes. The placement of the sample onto the deformation inducer, such that the sample is folded squarely in half, was crucial to the measurement and analysis. If the alignment was altered, several effects can occur. The surface area of the sample changes resulting in an error with respect to pressure determination, due to the assumption that the weight is placed up on a $3 \times 3 \mathrm{~cm}$ square of material. The second error occurs when the crease angle measurement is made, as the measurement technique assumes that the deformation occurs in the center of the material, with an equal mass of material on either side. If more or less material exists outside the sample holder the crease angle measurement will be erroneous, since the mass of the exposed sample affects the degree of resistance to the deformation (Skelton, 1968, p. 271). These errors are another reason for the multiple sample technique.

The transfer of the sample from the deformation inducer to the creasometer must be done in a very strict routine manner. Smooth motions and speed are required. The creased area should not be touched once the pressure 
weight has been removed (Raes et al., 1974). Any unnecessary handling could alter crease angle values. At low temperatures and pressures the deformation changes quickly and is vulnerable to any forces it experiences subsequent to the deformation process.

Therefore, the time for transfer must be reasonably constant for all samples; otherwise, some deformations will decay more than others. This is one of the reasons for the use of the creasometer rather than the set of crease standard wedges. Crease angle readings must also be made quickly, since decay of the deformation can be on the order of $1^{\circ} \mathrm{s}^{-1}$.

\section{Crease Measurements}

Once the sample was placed within the creasometer apparatus the crease angle could be determined. Care was taken to ensure that the base of the material was in-line with the alignment rod and that measurements were made along the flat surface of the alignment rod. In many cases the samples were observed to have a curvature. There were two apparent forms of curvature noticed. Raes et al. (1974) First was a bowing from top to bottom, resulting in the center of the sample being at a higher crease angle than the base. In this case the measurement was made with respect to the highest valued point. The second form involved a twisting of the base so that the front and back of the base corresponded to different values. In this case the measurement was made with respect to the center of the twist. Taking these errors into account and recording the variations possible it was determined that crease angle measurements were valid to an accuracy of $\pm 5^{\circ}$.

\section{Environmental Condition of Experiment}

Although temperature and pressure can be readily controlled, it was not possible to control the humidity of the apparatus. A general discussion of the effects of humidity is presented in the Appendix. An attempt to mitigate the influence of humidity was accomplished by restricting experiments to certain atmospheric conditions. Humidity levels were recorded for a period of time and the most frequent level was chosen as desirable. A level of $\ll 20 \%$ RH was observed quite often in the laboratory's test room. Therefore, measurements were made when the humidity was within this region. It was hoped that, given this restricted range of humidity fluctuation, the effects would be relatively continuous and minimal.

\section{Analysis of Results}

The aim of this investigation was to study the effects of time, temperature and pressure on the deformation response of blended-fiber materials, using a wool/polyester blend as a basis. Data was taken over the temperature range of $25^{\circ} \mathrm{C}$ to $127^{\circ} \mathrm{C}$. The weights used to induce the deformation were $0.5 \mathrm{~kg}, 1 \mathrm{~kg}$ and $2 \mathrm{~kg}$, all being used on the $3 \times 3 \mathrm{~cm}$ folded sample.
These weights equate to pressures of 5.45, 10.9 and $21.8 \mathrm{kPa}$ respectively. The deformation times used were 5, 10 and $15 \mathrm{~min}$. Measurements were carried out on material samples at all combinations of these parameters. Due to the extreme conditions experienced by some of the samples, it was not possible to obtain meaningful data for all combinations, as was explained previously. Large statistical errors were also present at very low temperatures. For these reasons, the data set range was narrowed so that only temperatures between $30^{\circ} \mathrm{C}$ and $110^{\circ} \mathrm{C}$ were considered. Only this data is used in the subsequent analysis.

An example of the obtained results in shown in Fig. 2a as a function of temperature and pressure for a given experimental time. This figure shows resulting crease angle for 3 pressure configurations, each as a function of varying temperature, for a deformation induction time of $5 \mathrm{~min}$. Observation of the data shows that temperature has a near linear relationship to crease angle, with an increase of $1{ }^{\circ} \mathrm{C}$ producing an average deformation increase of $0.8^{\circ}$. A least-squares linear fit for each of the three datasets is also shown, highlighting the general uniform increase in crease angle with increasing pressure. Similar trends were observed for other time conditions, with an obvious trend in the crease angle measurements corresponding to an increase in crease angle as the deformation time is increased.

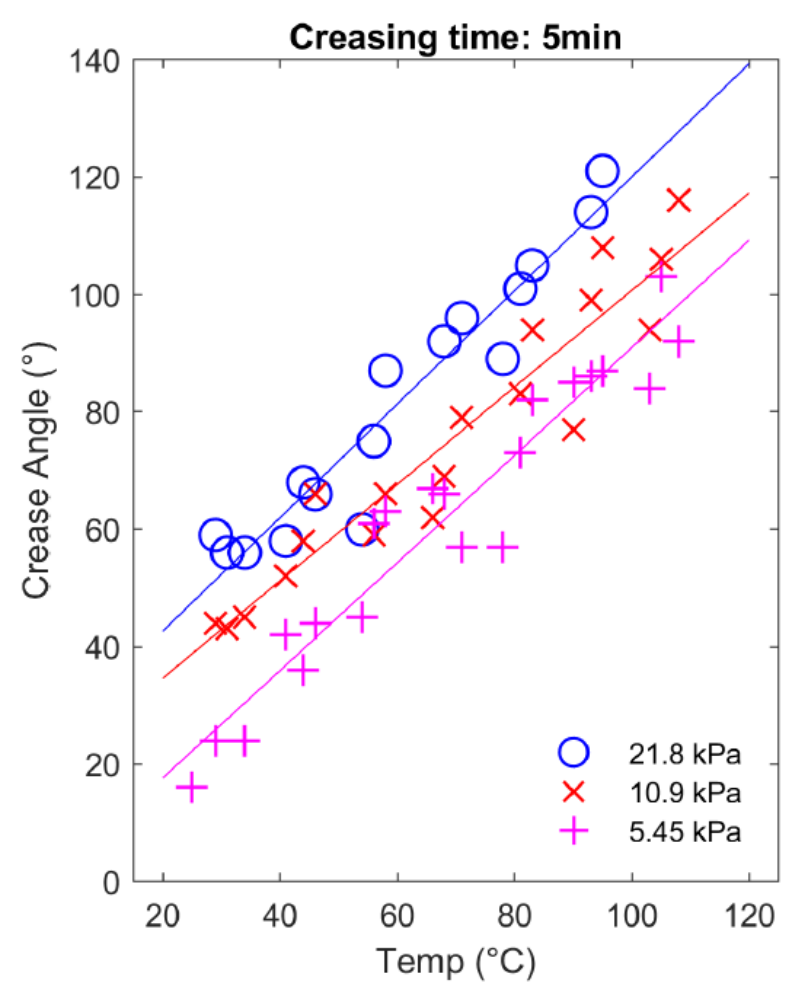

(a) 


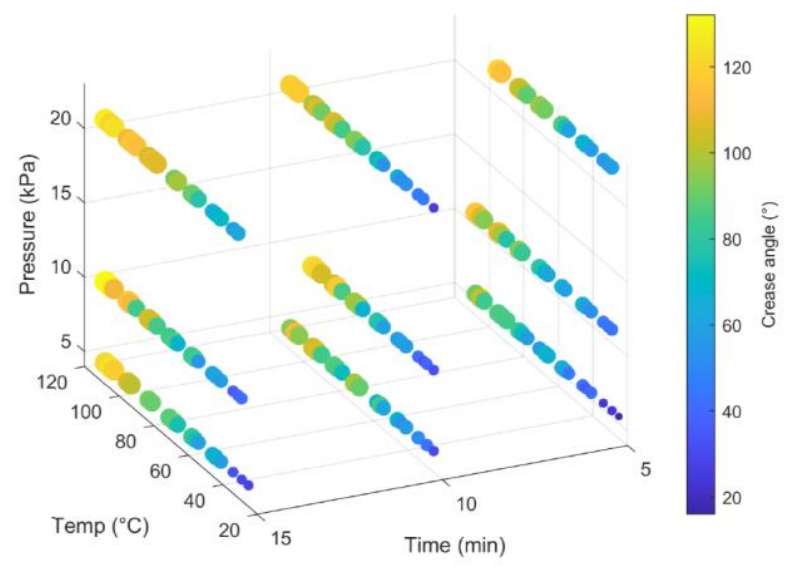

(b)

Fig. 2: Data results. (a) Example of crease angle as a function of temperature, $\mathrm{T}$, for a given deformation time, $(\mathrm{t}=5 \mathrm{~min})$, for 3 pressure conditions, with associated linear regressions. (b) Crease angle data space resulting from all measures as a function of the three control parameters (time, temperature and pressure)

There were, however, some data sets that exhibited the behavior described by Ranganathan et al. (1971), noting an increase in the crease angle between 50 and $70^{\circ} \mathrm{C}$. This tendency is observed here for the $\mathrm{p}=10.9 \mathrm{kPa}$ condition, though only slightly. Their experiments were performed on a cotton/polyester blend rather than a wool/polyester blend as done here. In addition, Ranganathan et al. (1971) reported data consisted of only 5 points. Therefore, only a similarity can be expected between results.

The full measurement data matrix set is shown in Fig. 2b, where each mean measurement result is plotted as a function of time, temperature and pressure according to the associated crease angle color map. While detailed inspection of this 4-D data is difficult, the full dataset can be used to derive a predictive model of crease angle as a function of test conditions. Given the general linear behavior observed, a linear regression model was proposed (using the fitlm function in Mat Lab) of the following form: Crease angle $=\alpha T+\beta \mathrm{t}+\gamma p+c$, where $\boldsymbol{T}$ is Temperature in ${ }^{\circ} \mathrm{C}, \boldsymbol{t}$ is time in min and $\boldsymbol{p}$ is pressure in $\mathrm{kPa}$. The resulting linear model of $\alpha=0.96, \beta=1.09, \gamma=$ 0.83 and $c=-6.37$ exhibits a root-mean-squared (rms) error of $9.47^{\circ}$ with a fit quality adjusted $R^{2}=0.874$. The inclusion of quadratic and cross terms (i.e., $\mathrm{T}^{2}$ or $\mathrm{Tt}$ ) reduced the rms error to $8.92^{\circ}$ for predicting crease angle, with an $\mathrm{R}^{2}=0.888$. Such added complexity is not considered necessary considering the minimal improvement in predictive quality.

Alternatively, an approximation of the linear model to Eq. (1) results in only a slight increase of the RMS error in crease angle of $9.7^{\circ}$. Considering the inherent variance is the experiment, this basic model seems suitable for general predictions:

crease_angle $\left({ }^{\circ}\right) \approx T\left({ }^{\circ} \mathrm{C}\right)+t(\min )+0.8 p(k P a)-6$

\section{Conclusion}

The effects of time, temperature and pressure as they pertain to induced crease deformations of a blended-fiber material have been investigated. A case study of the measurements of the crease induced in a wool/polyester fabric (45/55 mixture) was conducted using methodical means in an attempt to obtain quantitative results. The wool/polyester blend was chosen because of its greater resistance to deformation as related to other fibers and materials.

The results reported in this study document the effects of these parameters on deformations as measured through the crease angle. On the basis of the experimental results and the interpretations made during the analysis, a linear predictive model was developed which presents a roughly linear 1:1 relationship between the resulting crease angle and temperature, time and pressure variations due creasing. Results of the predictive model (RMS error of $9.7^{\circ}$ ) and a measurement repeatability error of $\pm 5^{\circ}$ can be compared to the model of Skelton (1968), which reported crease recover angle prediction accuracy of $10 \%$ for polyester fiber materials. Considering crease angle as $180^{\circ}$-crease_recovery_angle and a typical crease angle in the current study of $80^{\circ}$, a $10 \%$ error in c.r.a. would equate to $10^{\circ}$. Direct comparisons to actual data from Skelton (1968) are not possible, due to the difference in protocols (deformation time of $1 \mathrm{~min}$ followed by a relaxation time of the same). In addition, as said study focused on material differences, not deformation conditions, further comparison was not possible.

Further research could be carried out to examine the variation in the predictive model as a function of material details, be it composition, structural weave variations, or thread orientation. Such future studies could also be carried out under more controlled humidity conditions, though this constraint requires a significantly more complicated testing environment.

Due to the similar sensitivity to different test condition variables, one could imagine employing the results of this study to propose less energy consuming devices, where increased time and pressure are used to compensate for more energy inefficient heating components, to arrive at the same final crease performance result. The benefits of such an approach could be considered as a function of processing throughput and equipment costs associated with additional pressure constraints versus electrical costs for thermal treatment and general associated environmental impact. 


\section{Acknowledgment}

This article is dedicated posthumously to Prof. Brian Smith, University of Sussex, Brighton, whom without which this research project would not have been carried out.

\section{Ethics}

This article is original and contains unpublished material. The corresponding author confirms that all of the other authors have read and approved the manuscript and no ethical issues involved.

\section{References}

Abbott, N. J. (1965). Laboratory Evaluation of Wrinkle Recovery. Textile Research Journal, 35(3), 197-213. doi.org/10.1177/004051756503500301.

Choudhury, A. K. R. (2017). Principles of textile finishing. Woodhead Publishing. Textile Manufacturing, Woodhead Publishing Series in Textiles, pp, 363-427. Woodhead Publishing. doi.org/10.1533/9780857095633.3.363

Fan, J., \& Hunter, L. (2009). Engineering apparel fabrics and garments. Elsevier. doi.org/10.1533/9781845696443.52

Gray, B. (1992). Quantitative analysis of deformation conditions in blended fibres. MSc, University of Sussex, Brighton.

Hunter, L., \& Fan, J. (2015). Adding functionality to garments. In Textiles and fashion (pp. 705-737). Woodhead Publishing. doi.org/10.1016/B978-1-84569-9314.00028-3

Kärrholm, E., Bostwick, C., Silen, G., \& Kahlson, C. (1971). In Applied Polymer Symp, volume 18, page 999. Katz, H. (1966). Wrinkling Behavior of Wool Fabrics: Part I: A Multiple-Pleat Test. Textile Research Journal, 36, 874-887. doi.org/10.1177/004051756603601004

Kojima, M. (1986). U.S. Patent No. 4,623,770. Washington, DC: U.S. Patent and Trademark Office.

Leeder, J. (1975). Studies of Wrinkling Properties of Wool Fabrics: Part V: A Simplified Approach to the Testing of Wool and Wool-Rich Materials. Textile Research Journal, 45(8), 581-585. doi.org/10.1177/004051757504500802

Loasby, G., Mims, N. M., \& Rossiter, E. D. (1956). The construction and finishing of fabrics based on terylene polyester fibre in relation to crease-recovery. Journal of the Textile Institute Proceedings, 47(8), P708-P723. doi.org/10.1080/19447015608665311

Looney, F. S. J., \& Handy, C. (1968). The effects of construction on the wear wrinkling of Dacron®/wool suitings. Textile Research Journal, 38, 989-998. doi.org/10.1177/004051756803801003
Ly, N. G., \& Denby, E. F. (1983). Bending strain in a sharp fabric crease. Textile Research Journal, 53(9), 571-573.

Mercer, H. L. and Moore, C. T. (1975a). Method of increasing the crease resistance of cellulosic fibres.

Moore, C. T., \& Mercer, H. L. (1975b). U.S. Patent No. 3,883,300. Washington, DC: U.S. Patent and Trademark Office.

Militký, J., Kryštůfek, J., \& Hartych, V. (1991). Modified polyester fibres. Elsevier Science Publishers, PO Box 211, 100 AE Amsterdam, The Netherlands. https://dspace.tul.cz/handle/15240/16554

Postle, R., \& Carnaby, G. A. (1988). S. de long, 'The Mechanics of Wool Structures'.

RAES, G. (1974). Crease recovery angles of fibre bundles. influence of chemical treatments. https://pascal-

francis.inist.fr/vibad/index.php?action=getRecordDe tail\&idt=PASCAL7578005903

Ranganathan, S. R., Elder, H. M., \& McLaren, I. F. (1971). Effect of moisture and temperature on creaserecovery of cotton fabrics and blended-fibre fabrics containing cotton. 2. Crease-recovery performance of resin-treated cotton and polyester-fibre-cotton fabrics in different environments. JOURNAL OF THE TEXTILE INSTITUTE, 62(7), 393-+.Roy

Choudhury, A. (2013). Process control in finishing of textiles. In Majumdar, A., Das, A., Alagirusamy, R. and Kothari, V., editors, Process Control in https://www.sciencedirect.com/science/article/pii/B9 7808570902705001

Ravandi, S. H., \& Valizadeh, M. (2011). Properties of fibers and fabrics that contribute to human comfort. In Improving comfort in clothing (pp. 61-78). Woodhead Publishing. doi.org/10.1533/9780857090645.1.61

Skelton, J. (1968). 22-a theoretical and experimental investigation of the crease recovery of plain-weave fabrics woven from staple yarns. Journal of the Textile Institute, 59(6), 261-284. doi.org/10.1080/00405006808659986

Smuts, S. (1989). A Review of the Wrinkling of Wool and Wool/polyester Fabrics. South African Wool and Textile Research Institute. doi.org/10.1177/004051755902900806

Wilkinson, P. R., \& Hoffman, R. M. (1959). The Effects of Wear and Laundering on the Wrinkling of Fabrics1. Textile Research Journal, 29(8), 652-660. doi.org/10.1177/004051755902900806

\section{Appendix: Humidity}

Some work has been carried out concerning the effects of varying humidity and moisture content of materials and analyzing the deformation responses. It seems though that there are conflicting results from various researches. The effects of humidity have been assumed to be important 
and were considered in the design of the experiment. It is therefore important to report the findings and precautions taken by other researchers. Leeder (1975) showed that fabrics containing an excess of 55\% polyester were relatively unaffected by humidity with regards to deformation recovery. Experiments were carried out over the full range of moisture content, 0 to $100 \% \mathrm{RH}$. The work was all performed from 20 to $35^{\circ} \mathrm{C}$.

Skelton (1968) showed similar effects, with the temperature range being expanded to $-10^{\circ} \mathrm{C}$ to $110^{\circ} \mathrm{C}$. When he began making higher temperature measurements $\left(35^{\circ} \mathrm{C}\right)$, he estimated that the effects of humidity would be reduced, due to the increased evaporation and stated that "the effect of a rise in humidity on the crease-recovery of a polyester-fiber fabric is likely to be small, at least until the humidity approaches $80 \%$." (Ranganathan et al., 1971 , p. 398) It was also stated that experimental interpolations were possible given "the humidity is low (22 to $0 \% \mathrm{RH}$ ) provided that the effect of moisture is ignored as a first approximation. Ranganathan et al. (1971, p. 400) This agreed with Leeder with the addition of a broader temperature range.

In strict contrast to the previous results is that of Kärrholm et al. (1971) and deformation recovery research as pertaining to humidity. Katz claimed that climatic conditions are probably the most important factor in deformation experiments. The effects are further modified by the specific fibers size, weave, density and absorbency. Katz showed that variations of humidity could change the crease angle and that this change increased with temperature. A shift from 65 to $75 \% \mathrm{RH}$ at $20^{\circ} \mathrm{C}$ resulted in a crease angle deviation of $5 \%$, while the experiment at $40^{\circ} \mathrm{C}$ showed a change of $10 \%$. According to Katz, "climatic conditions... are far more important than those of fabric construction and the importance of controlling the level of humidity... cannot be over-emphasized" (Smuts, 1989, p. 37) and (Kärrholm et al., 1971). Measurements were conducted on a multiple deformation apparatus within a hermetically sealed enclosure.

The variations on experimental conditions have prompted the need for standardizations within deformation testing. Only if test conditions are performed under constant humidity conditions can direct relations between tests be expected. It is not possible to correlate data from different experiments if the conditions are not the same. It has been suggested by several research groups to introduce a set condition for certain experiments (Wilkinson and Hoffman, 1959) and (Smuts, 1989, p. 41).

This condition corresponds to deformations at $32^{\circ} \mathrm{C}$ and $85 \% \mathrm{RH}$, with measurements made at $21^{\circ} \mathrm{C}$ and $65 \% \mathrm{RH}$. This idea of rigorous standardization has not been accepted by all research organizations as yet.

In the current study, one can hypotheses that the sample responds to humidity fluctuations by exhibiting larger deformations proportional to an increase in humidity (Abbott, 1965) and (Smuts, 1989, p. 37). This is due to the wool fibers opening up and swelling from the moisture. This makes them limper and more pliable (Mercer and Moore, 1975b). The effect being that deformations are induced quicker and more permanent given all other parameters equal. The use of steam was therefore acknowledged as providing an enhanced method for deformation alteration. As the temperature of the measurement was increased the humidity level was less important for the sample response, since it was being exposed to adequate degrees of heat thereby removing inherent moisture. When steam was required to remove any deformation a substantial amount of time was taken to remove the induced humidity via dry heat. 